\title{
Prismatic mesh generation based on anisotropic volume harmonic field
}

\section{Yiming Zhu}

Dalian University of Technology

\section{Shengfa Wang}

Dalian University of Technology

\section{Xiaopeng Zheng}

Dalian University of Technology

$\mathrm{Na}$ Lei

Dalian University of Technology

Zhongxuan Luo

Dalian University of Technology

Bo Chen ( $\nabla$ chenbo01010401@163.com )

Computational Aerodynamics Institute China Aerodynamic Research and Development Center

\section{Research}

Keywords: Mesh generation, Prismatic mesh, Volume harmonic eld, 3D tensor, Minkouski sum

Posted Date: January 21st, 2021

DOl: https://doi.org/10.21203/rs.3.rs-146632/v1

License: (c) (i) This work is licensed under a Creative Commons Attribution 4.0 International License. Read Full License

Version of Record: A version of this preprint was published at Advances in Aerodynamics on May 10th, 2021. See the published version at https://doi.org/10.1186/s42774-021-00065-y. 


\section{Abstract}

In this paper, we present an effective prismatic mesh generation method for viscous flow simulations. To address the prismatic mesh collisions in recessed cavities or slit areas, we exploit 3D tensors controlled anisotropic volume harmonic field to generate prismatic meshes. Specially, a well-fitting tetrahedral mesh is first constructed to serve as the discrete computation domain of volume harmonic fields. Then, 3D tensors are exploited to control the volume harmonic field that better fits the shape geometry. From the topological perspective, the generation of the prismatic mesh can be treated as a topology-preserved morphing of the viscous wall. Therefore, iso-surfaces in the volume harmonic field should be homeomorphic to the viscous wall while fitting its shapes. Finally, a full prismatic mesh can be induced by estimating the forward directions and visible regions in the volume harmonic field. Moreover, to be compatible with different simulation situations, the thickness of prismatic meshes should be variable. Our approach provides local adjustable thickness of prismatic meshes, which can be achieved by controlling local 3D tensors. The proposed anisotropic volume harmonic field based prismatic meshes are efficient and robust, and a full prismatic mesh can be guaranteed without low quality collisions. Various experiments have shown that our proposed prismatic meshes have obvious advantages in terms of efficiency and effectiveness.

\section{Full Text}

Due to technical limitations, full-text HTML conversion of this manuscript could not be completed. However, the manuscript can be downloaded and accessed as a PDF.

\section{Figures}




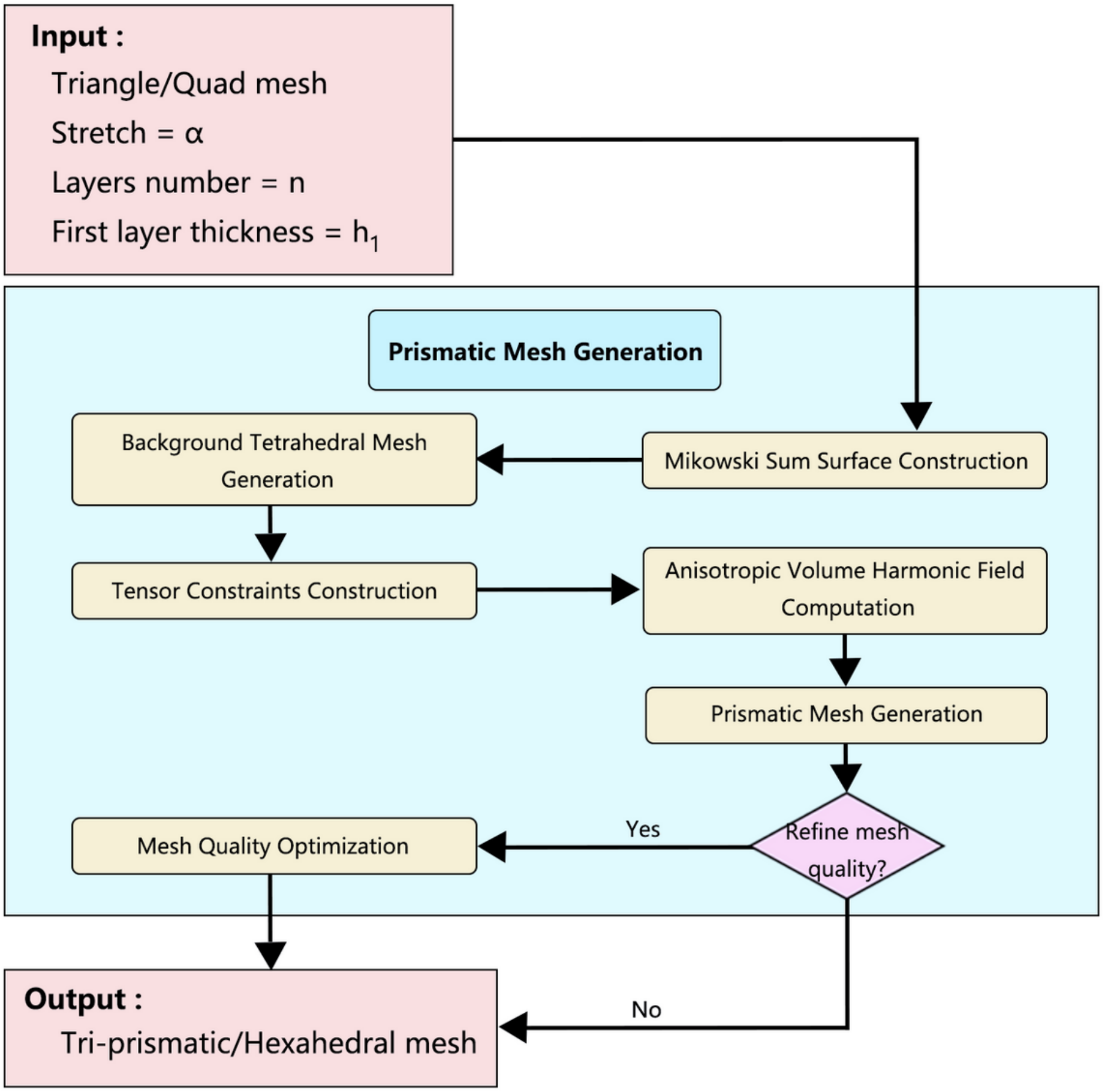

Figure 1

Prismatic mesh generation framework. 


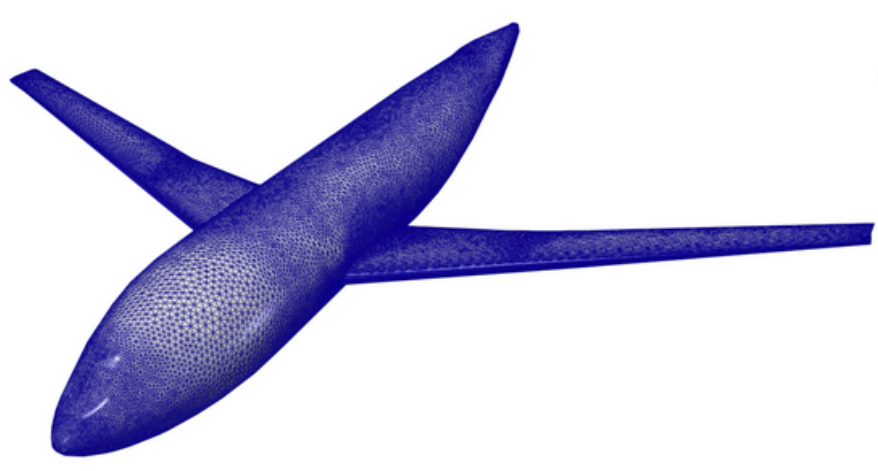

(a)

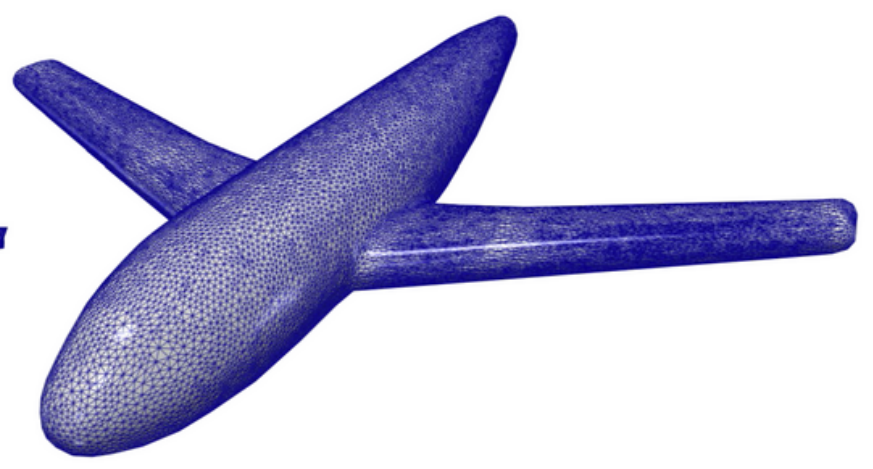

(b)

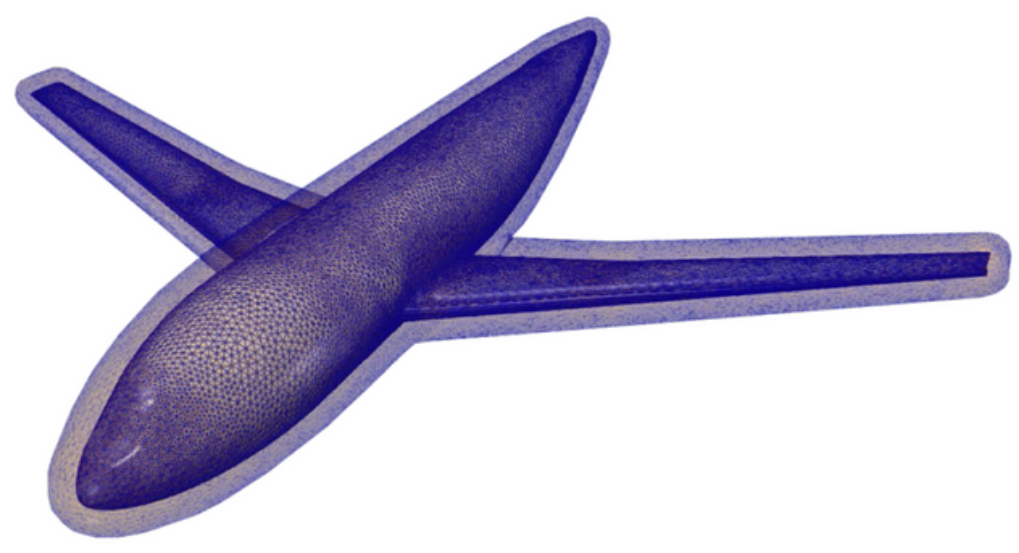

(c)

\section{Figure 2}

Illustration of the Minkowski Sum boundary surface (envelope surface). (a)The original surface mesh (viscous wall). (b) The Minkowski Sum boundary surface. (c) The combination of the Minkowski Sum boundary surface and the original surface. 

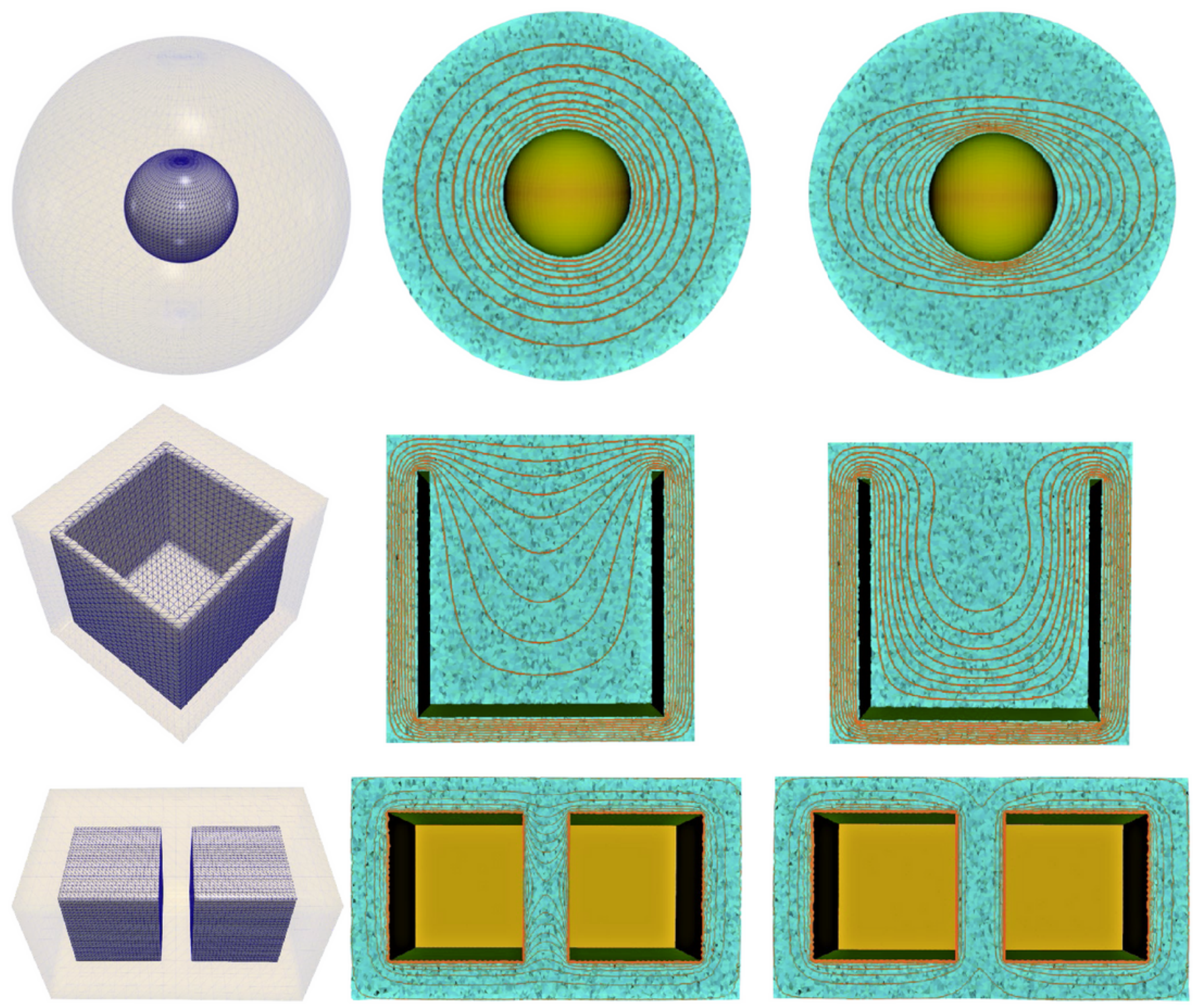

(a)

(b)

(c)

\section{Figure 3}

Illustration of the anisotropic volume harmonic field. (a) The original models with Minkowski Sum boundary surfaces; (b) The section of iso-surfaces induced by standard volume harmonic field; (c) The section of iso-surfaces induced by the proposed anisotropic volume harmonic field. 

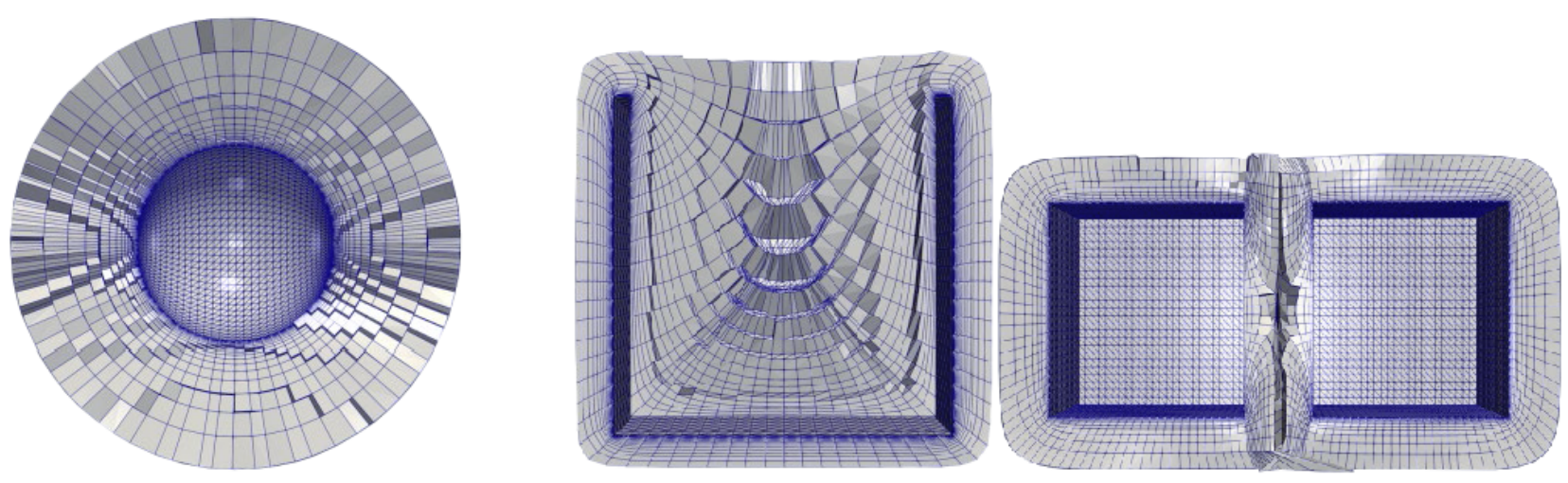

(a)
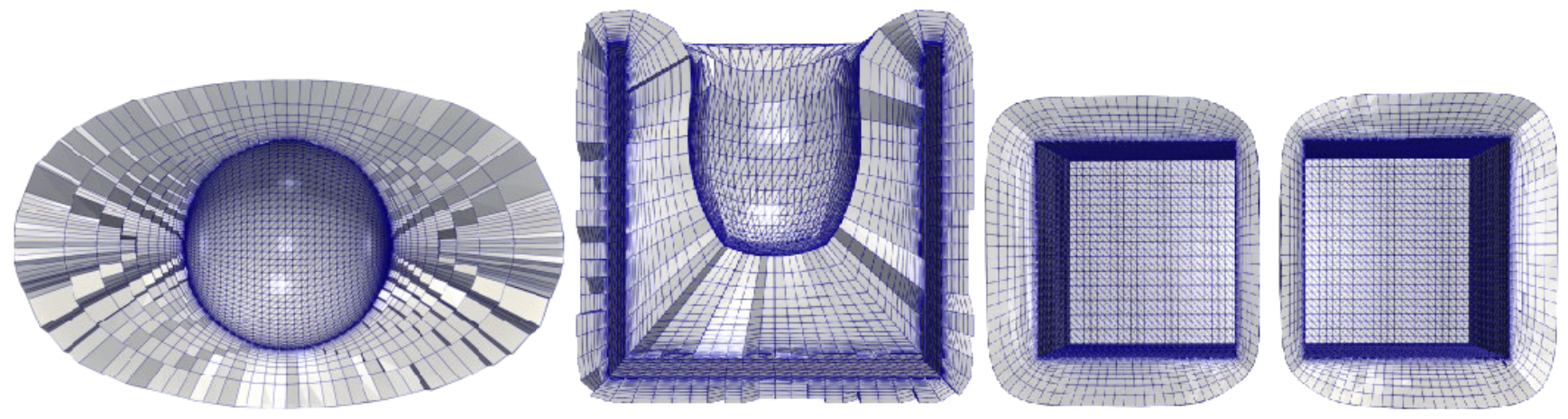

(b)

Figure 4

Illustration of the prismatic meshes. (a) The prismatic meshes induced by standard volume harmonic field; (c) The prismatic meshes induced by the proposed anisotropic volume harmonic field. 


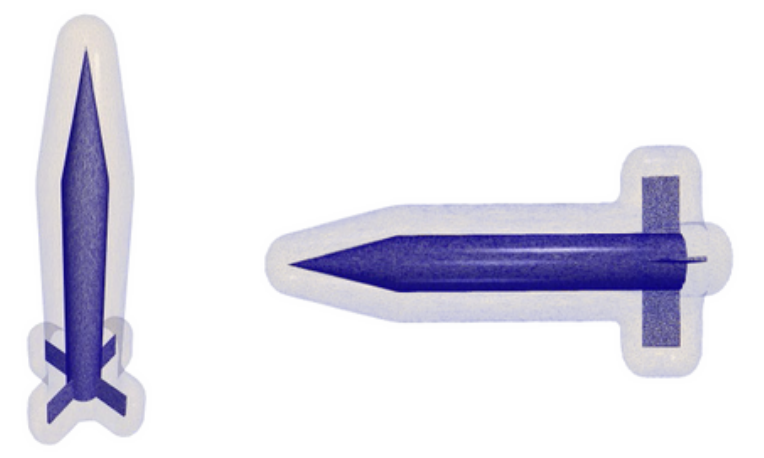

(a) Original model with Minkowski Sum boundary surface
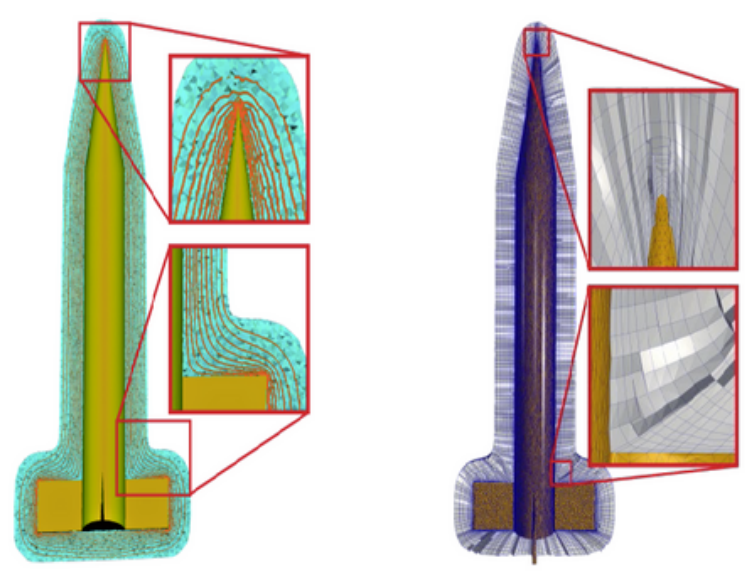

(b) The standard iso-surfaces

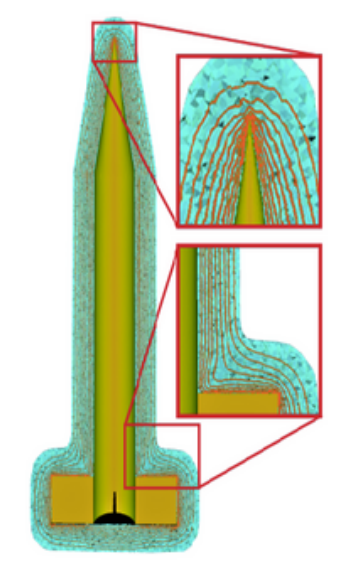

The standard prismatic mesh

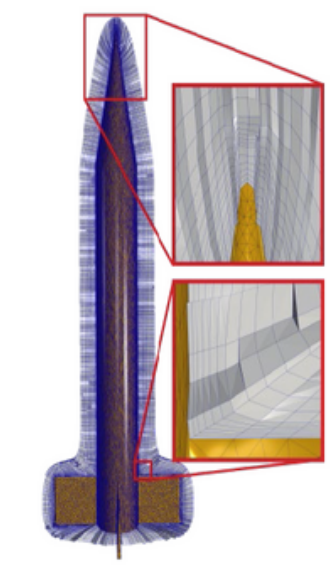

(c) The proposed iso-surfaces The proposed prismatic mesh

\section{Figure 5}

Comparison of prismatic meshes. (a)The original surface and envelope surface; (b) and (c) The isosurfaces and prismatic meshess generated using the standard harmonic field and the proposed anisotropic harmonic field, respectively. 

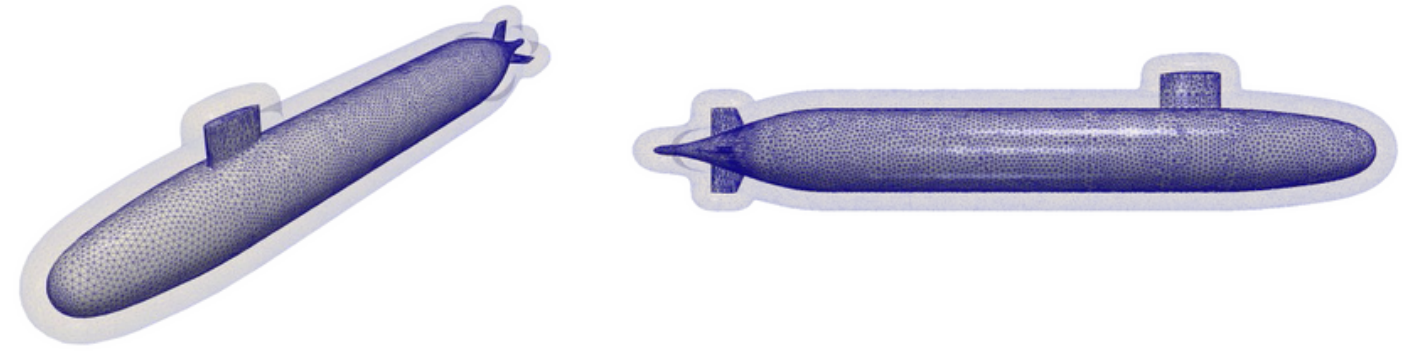

(a) Original model with Minkowski Sum boundary surface
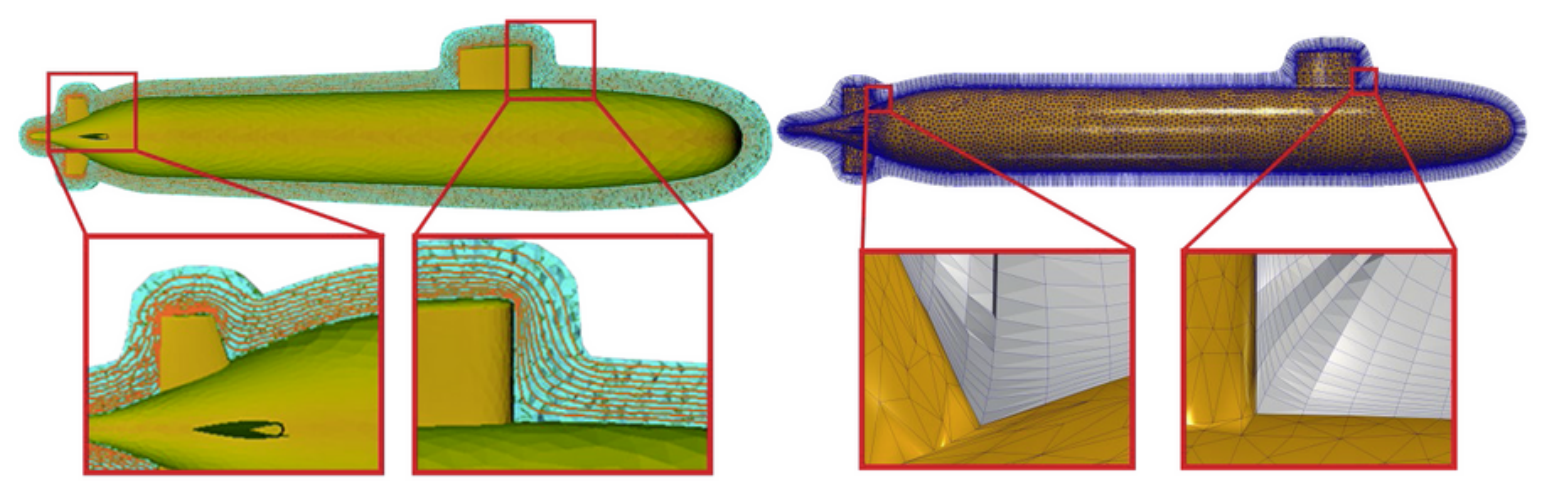

(b) The standard iso-surfaces The standard prismatic mesh

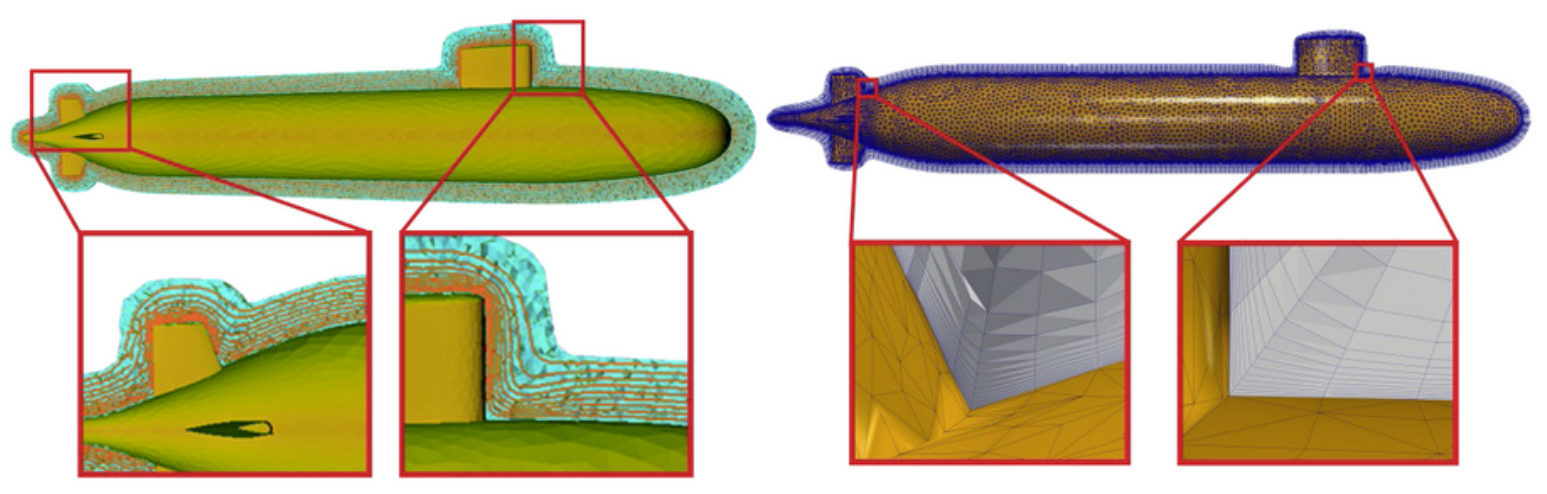

(c) The proposed iso-surfaces The proposed prismatic mesh

\section{Figure 6}

Prismatic mesh of submarine model. (a)The original surface and envelope surface; (b) The iso-surfaces and prismatic meshes generated using the standard harmonic field and the proposed anisotropic harmonic field, respectively. 

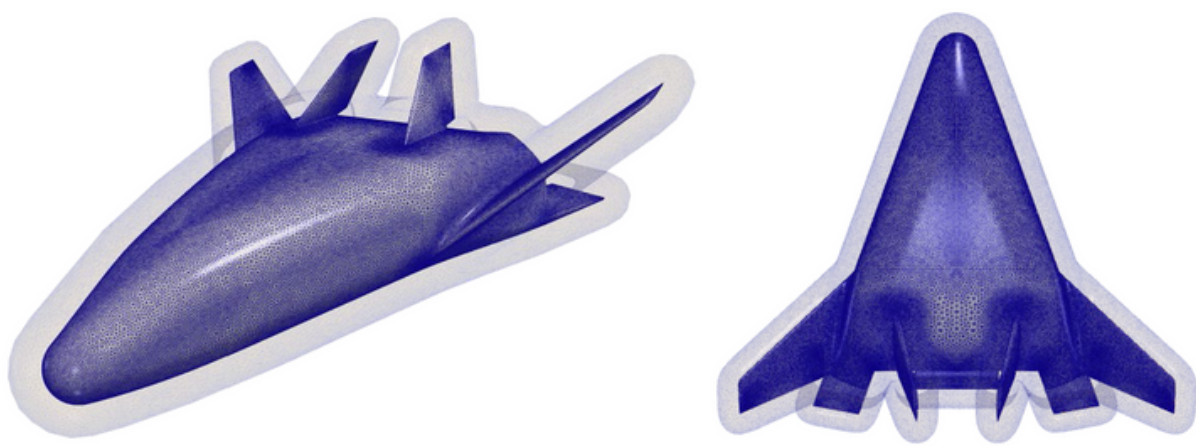

(a) Original model with Minkowski Sum boundary surface

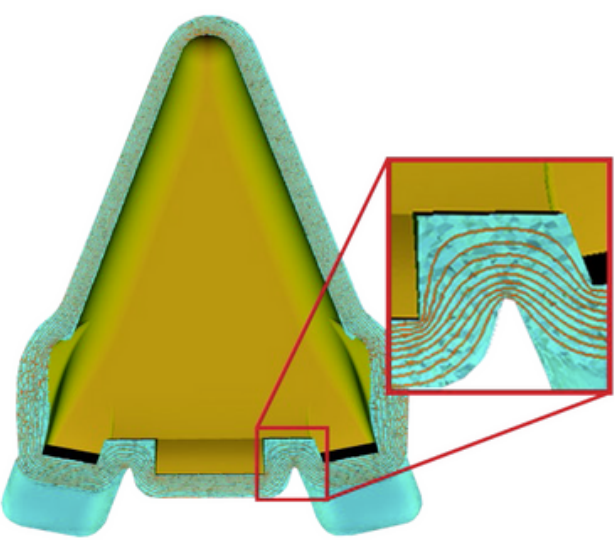

(b) The standard iso-surfaces

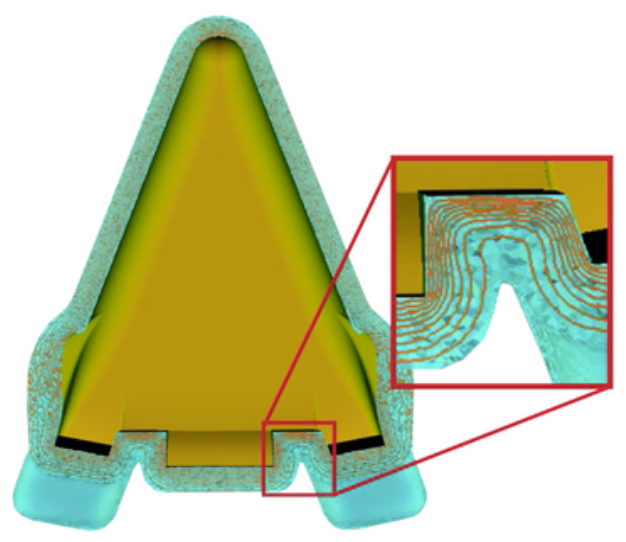

(c) The proposed iso-surfaces

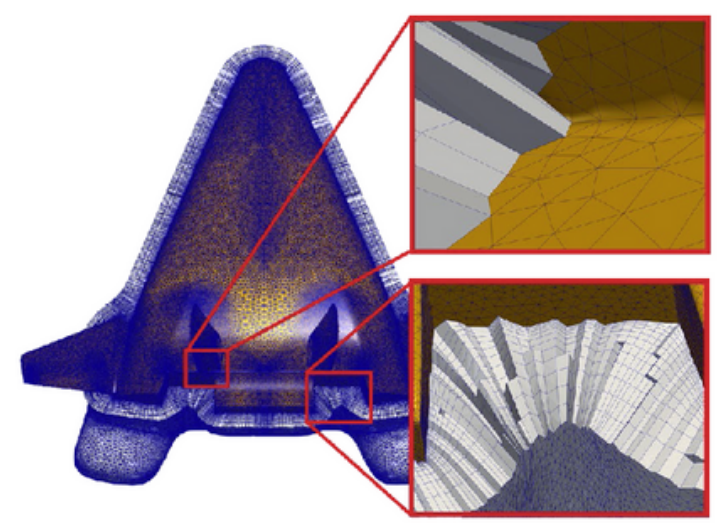

The standard prismatic mesh

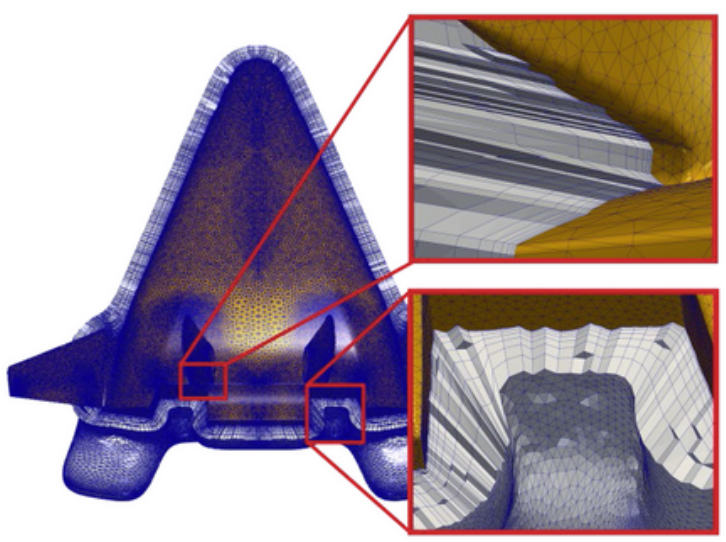

The proposed prismatic mesh

\section{Figure 7}

Prismatic mesh of unmanned aerial vehicle model. (a)The original surface and envelope surface; (b) The iso-surfaces and prismatic meshes generated using the standard harmonic field and the proposed anisotropic harmonic field, respectively. 


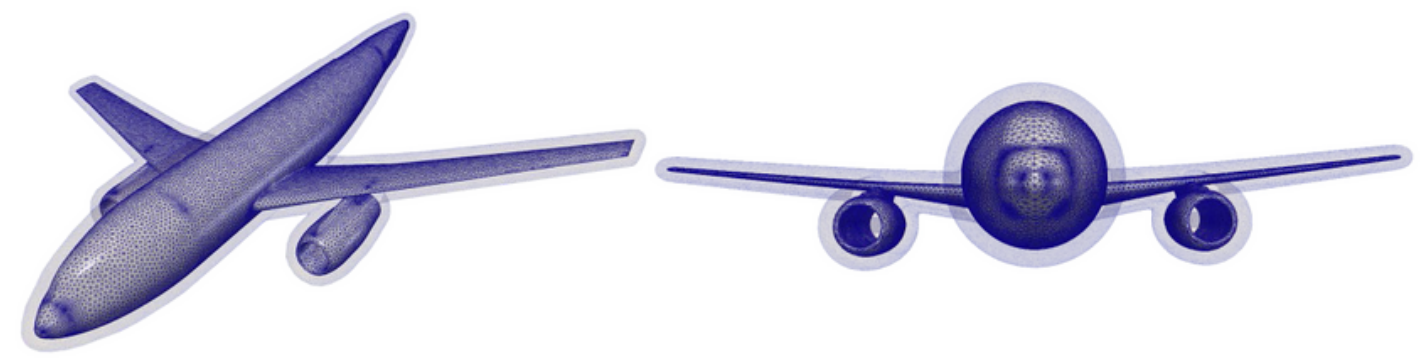

(a) Original model with Minkowski Sum boundary surface

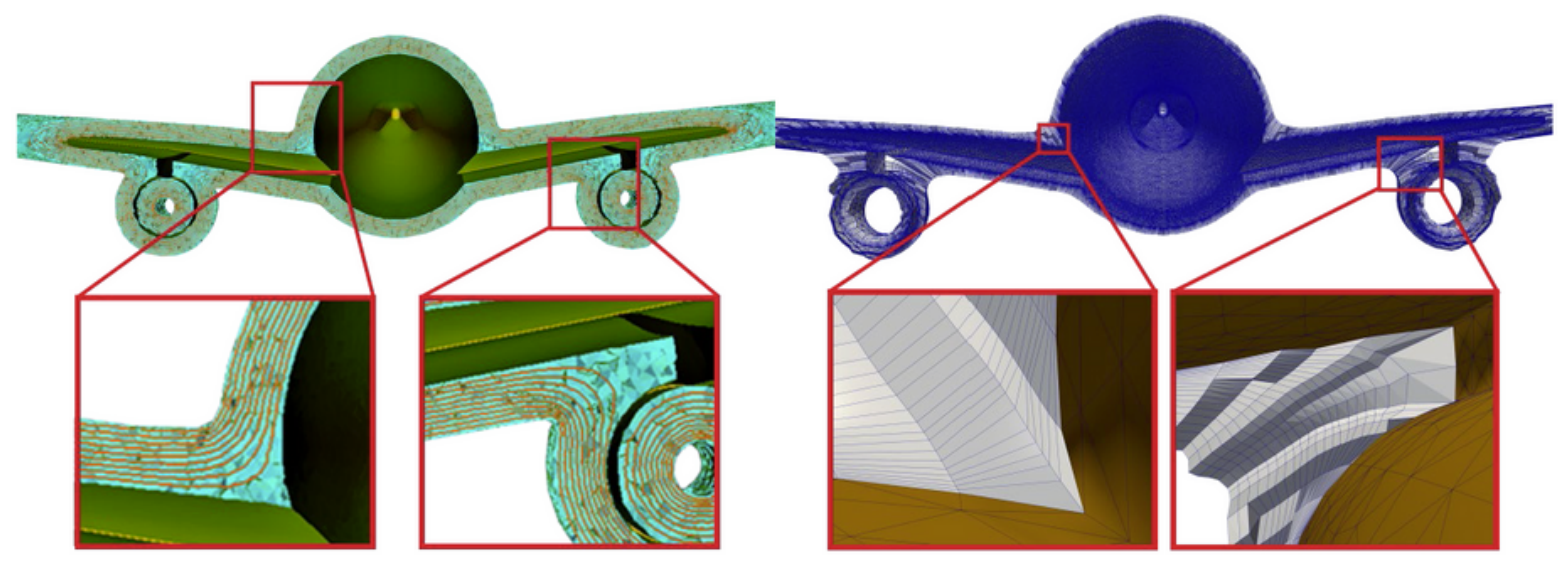

(b) The standard iso-surfaces

The standard prismatic mesh

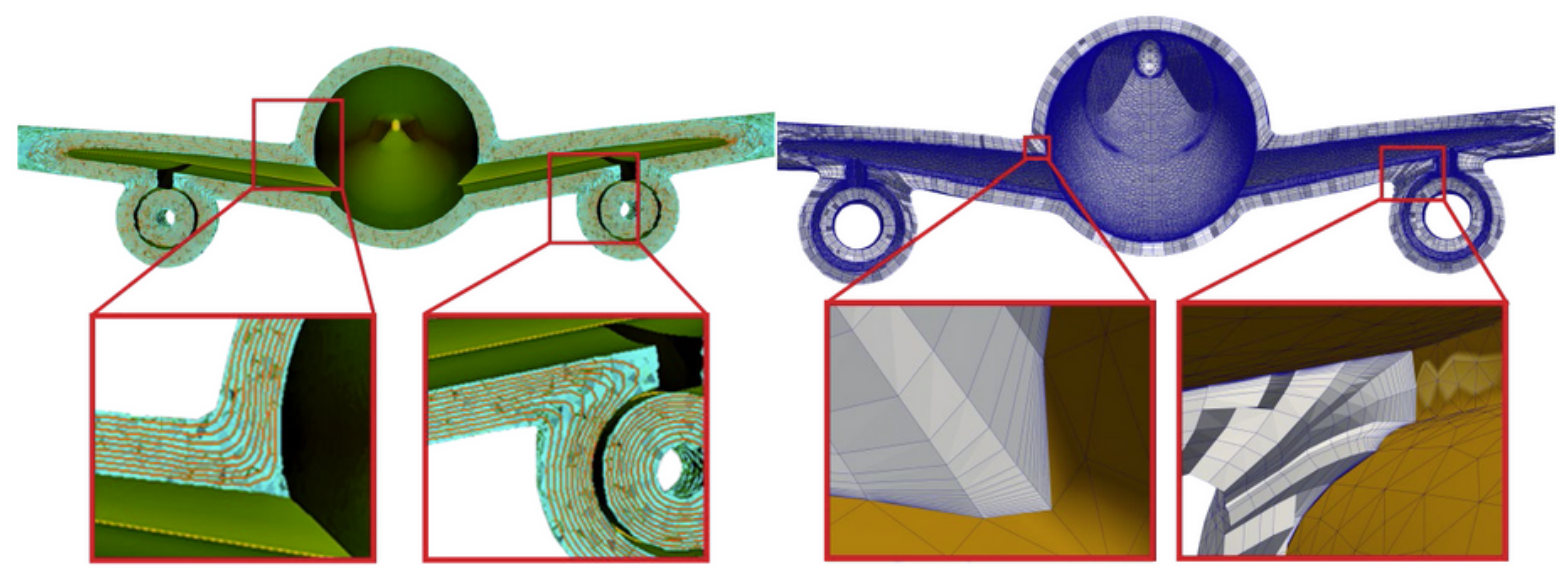

(c) The proposed iso-surfaces The proposed prismatic mesh

\section{Figure 8}

Prismatic mesh of plane model with complex geometries and genus. (a)The original surface and envelope surface; (b) The isosurfaces and prismatic meshes generated using the standard harmonic field and the proposed anisotropic harmonic field, respectively. 